\title{
关于模 $p^{l}$ 的元根 \\ 孙琦李鱰光 \\ (四川大学数学系, 成都)
}

最近，孙琦 ${ }^{[1]}$ 证明了有限域上有关元根的一个结果: 设 $p$ 是一个素数, $G \cdot F\left(p^{n}\right)$ 是一个. 含 $p^{n}$ 个元的有限域, $u, v, \theta$ 是 $G F\left(p^{n}\right)$ 中三个任给的非零元, 如果 $p^{n} \geqslant 2^{60}$, 则在 $G F\left(p^{n}\right)$ 中存在二个元根 $\alpha$ 和 $\beta$, 使得 $u \alpha+\nu \beta=\theta$. 在文献[1]中,作者对 $n=1, p$ 是奇素数的情 形,给出了详细的证明.

一个很自然的问题是: 对于模 $p^{l}(l \geqslant 2, p$ 是奇素数)的元根, 上述结果是否也成立?

本文回答了这一问题. 我们证明了以下的定理.

定理 设 $p$ 是一个奇素数， $l \geqslant 2$ 是一个整数, $a, b, c$ 是给定的整数, 满足 $p \nmid a b c$, 则当 $p>2^{\infty 0}$ 时, 至少存在 $(p-2) p^{l-1}$ 对模 $p^{l}$ 的元根 $\alpha$ 和 $\beta$ 使得

$$
a \alpha+b \beta \equiv c\left(\bmod p^{\prime}\right)
$$

成立.

在证明定理之前, 先证明下面的引理.

引理设 $p$ 是一个奇素数, $g$ 是模 $p$ 的一个元根, 则在数列

$$
g+i p, \quad i=0,1, \cdots, p-1
$$

中至少有 $p-1$ 个模 $p^{l}(l \geqslant 2)$ 的元根.

证 由于 $g+j p(j=0,1, \cdots, p-1)$ 均为模 $p$ 的元根, 热知, 当 $(g+i p)^{p-1 \neq}$ $l\left(\bmod p^{2}\right)$ 时, 则 $g+i p$ 为模 $p^{l}(l \geqslant 2)$ 的元根. 显然，(2)式中任意两个数对模 $p^{l}$ 均不同 余. 因此,如果我们能够证明(2)式中任意两个数的 $p-1$ 次方幂对模 $p^{2}$ 均不同余, 便证明了 引理. 设 $0 \leqslant i_{1}<i_{2} \leqslant p-1$, 如果

$$
\left(g+j_{1} p\right)^{p-1} \equiv\left(g+j_{2} p\right)^{p-1}\left(\bmod p^{2}\right)
$$

可得

由此推出

$$
g^{p-1}+g^{p-2}(p-1) j_{1} p \equiv g^{p-1}+g^{p-2}(p-1) j_{2} p\left(\bmod p^{2}\right)
$$

$$
i_{1} \equiv i_{2}(\bmod p),
$$

此不可能. 这就证明了(2)式中至少有 $p-1$ 个数, 它们的 $p-1$ 次方害模 $p^{2}$ 均不与 1 同余. 证完.

定理的证明:

由文献 [1]的结果知, 存在模 $P$ 的二个元根 $g_{1}, g_{2}$, 使同余式

$$
a g_{1}+b g_{2} \equiv c(\bmod p)
$$

成立. 设 $a g_{1}+b g_{3}-c+k p, k \in Z$, 考虑同余式 


$$
a\left(g_{1}+t p\right)+b\left(g_{2}+s p\right) \equiv c\left(\bmod p^{2}\right),
$$

其中 $0 \leqslant t \leqslant p-1,0 \leqslant s \leqslant p-1$, 将 $a g_{1}+b g_{2}=c+k p$ 代入(3)式, 即得

$$
k+a t+b s \equiv 0(\bmod p), \quad 0 \leqslant t, s \leqslant p-1,
$$

显然, (4) 式有 $P$ 组解 $\left(t_{1}, s_{1}\right),\left(t_{2}, s_{2}\right), \cdots \cdots,\left(t_{p}, s_{p}\right)$, 其中 $s_{1}, \cdots, s_{p}$ 和 $t_{1}, \cdots, t_{p}$ 分别 为 $0,1, \cdots, p-1$ 的某一排列. 于是由引理知, 至少有 $p-2$ 对 (4)式的解, 不妨设为 $\left(t_{i}\right.$, $s_{i}$ ), $i-1, \cdots, p-2$, 使 $g_{1}+t_{i} p$ 和 $g_{2}+s_{i} p$ 均为模 $p^{2}$ 的元根（它们的 $p-1$ 次方等模 $p^{2}$ 均不与 1 同余), 这就证明了定理对 $l=2$ 成立.

现设定理对 $l=t$ 成立 $(f \geqslant 2)$, 即有 $(p-2) p^{t-2}$ 对模 $p^{f}$ 的元根 $\alpha_{i}, \beta_{i}$, 且 $\alpha_{i}^{p-1} \neq$ : $\left(\bmod p^{2}\right), \beta_{i}^{p-1} \neq 1\left(\bmod p^{2}\right),\left(j-1, \cdots,(p-2) p^{1-2}\right)$, 使

$$
a \alpha_{i}+b \beta_{i} \equiv c\left(\bmod p^{\prime}\right)
$$

成立.

现在,我们来证明定理对 $l=f+1$ 也成立. 由(5)式可设 $a \alpha_{i}+b \beta_{i}-c+k_{1} p^{\prime}, k_{1} \epsilon$ $z$, 考虑同余式

$$
a\left(\alpha_{j}+c_{i} p^{\prime}\right)+b\left(\beta_{i}+c_{i}^{\prime} p^{\prime}\right) \equiv c\left(\bmod p^{\prime+1}\right),
$$

其中 $0 \leqslant e_{j} \leqslant p-1,0 \leqslant c_{i}^{\prime} \leqslant p-1$, 将 $a \alpha_{j}+b \beta_{i}=c+k_{1} p^{\prime}$ 代入(6)式, 即得

$$
k_{1}+a e_{j}+b e_{i}^{\prime} \equiv 0(\bmod p), \quad 0 \leqslant e_{j}, e_{i}^{\prime} \leqslant p-1,
$$

显然,同余式(7)有 $p$ 组解 $\left(e_{i_{0},}, e_{i_{0}}^{\prime}\right), t-1, \cdots, p$, 其中 $e_{i, 1}, \cdots, c_{i, p}$ 和 $e_{i}^{\prime}, \cdots, c_{i, p}^{\prime}$ 分 别为 $0,1, \cdots, p-1$ 的某一排列, 且每一组解 $\left(e_{i, s}, e_{i, s}^{\prime}\right)$ 使 $\alpha_{j}+e_{i, s} p^{\prime}, \beta_{i}+c_{i, s}^{\prime} p^{\prime}$ 均为模 $p^{j+1}$ 的元根. 这就证明了共给出 $(p-2) p^{j-1}$ 对模 $p^{f+1}$ 的元根 $\alpha, \beta$ (其中任意两对对模 $p^{j+1}$ 是不同的)使

成立.

$$
a \alpha+b \beta \equiv c\left(\bmod p^{\prime+1}\right)
$$

\section{* 文 常}

(1) Sun Qi (孙), On primitive roots in a Finile field, 数学进展, 2(1987). 